\title{
Inconsistent Guard Time Span With on Mobile 4G System
}

\author{
Lway Faisal Abdulrazak ${ }^{2}$ \\ ${ }^{1}$ Computer Science Department, Cihan University Sulaimanyia, Iraq
}

\begin{abstract}
Guard time span (GT) is implemented as Cyclic Prefix (CP) to completely alleviate Inter-symbol Interference (ISI) and to preserve orthogonality among OFDMA subcarriers as long as the guard time span is sufficiently greater than channel delay spread. In conventional OFDMA systems GT span is fixed and chosen to be much longer to tolerate worst case condition irrespective of current propagation channel state. This technique, however, degrades the overall spectral efficiency as well as consumes transmitter energy proportional to the span of the guard time. This fact motivates the need to vary the guard time span based channel parameters especially for mobile applications. The primary goal of this paper is to vary the GT span based channel delay spread for mobile $4 G$ technology.
\end{abstract}

Keywords : Guard Time; Mobile 4G; OFDMA; Delay Spread.

\section{Introduction}

Mobile 4G is very new technology on horizon being developed under IEEE802.16e-2005 standard [1] to revolutionize broadband wireless access systems. This technology designed to provides interoperable broadband wireless connectivity to fixed, nomadic, portable and mobile users. Ultimately, it can provides lowcost, high speed data rates up to $75 \mathrm{Mbps}$ at vehicular speeds greater than $120 \mathrm{Km} / \mathrm{h}$ covering an area over a radius potentially of up to 30 miles without the need of direct line-of-sight (LOS). To accomplish these goals and to overcome the problems associated with multipath channel, mobile WiAMX uses essential features like OFDMA as the radio interface, adaptive modulation and Multi Input Multi Output (MIMO) technology [2].

Orthogonal Frequency Division Multiple Access (OFDMA) is a multicarrier transmission technique that extends OFDM for use as a multiple access technology, in which the available bandwidth is split into equidistant narrow band subchannels, each consisting of a set of subcarriers. For each subcarrier, the modulation and coding can be adapted separately. By virtue of its long symbol time and use of Guard time span OFDMA can effectively cope with the larger delay spread, thereby increasing the data throughput and minimizing the equalization process. Moreover, OFDMA presents a number of advantages such as high spectral efficiency, resilience to Radio Frequency (RF) interference and lower multipath distortion, which make it an attractive choice for next generation wireless communication systems, commonly referred to beyond 3G (B3G) [3].

Guard time span is one of the key OFDMA parameters. This span is a copy of the last portion of the useful symbol time appended to the beginning of each transmitted symbol to completely alleviate Intersymbol Interference (ISI) as long as the GT is greater than delay spread of the channel. By implementing the GT as cyclic prefix (CP) the system being immune to Intercarrier Interference (ICI) that causes a severe degradation of Quality of Service (QoS) in OFDMA systems. In addition, the CP span has advantage of allowing perfect channel estimation as well as timing and frequency synchronization [4]. Conventional OFDMA system uses large-static guard time span to tolerate worst case channel condition irrespective of current channel state. This technique, however, causes a loss in bandwidth efficiency as well as waste transmitter energy. The amount of power wasted depends on how large a fraction of the OFDMA symbol duration the guard time is. By using Inconsistent GT span, significant improvement in data throughput can be obtained specifically under ideal or moderate channel conditions whereas mobile user nearby base station or passing indoor environments. Since phones need to run on battery, optimization provides the ability to reliably send information at the lowest possible power level, which has advantage of extending the battery life of mobile devices.

In this paper we address the influence of varying guard time span on mobile $4 \mathrm{G}$ system performance operating on mutipath fading channel. We use disparate spans to investigate the resultant packet error rate (PER) used to describe the transmission channel quality. In the simulation, we use the standard mobile $4 \mathrm{G}$ parameters specified in [5]. The rest of this paper is organized as follows. In section 2, we describe briefly the general concept of OFDMA systems. In particular, section 2.1 and 2.2 gives general overview about guard time optimization and delay spread measurements, respectively. Section 3 shows how to calculate data rates and loss in SNR due to guard time insertion. In Section 4, simulation results are presented, while section 5, discuss the conclusion. 


\section{System Discription}

The block diagram of OFDM transceiver based mobile 4G system is shown in Figure 1. The serial $\boldsymbol{k}$ input binary bits are first forward error encoded (FEC), punctured and interleaved to allow detection and correction of errors that may occur during signal transmission. After encoding, the $\boldsymbol{n}$ coded bits are mapped to a sequence of complex data symbols. Symbols are further grouped to form transmitted frames, each with $N$ symbols. For OFDMA, the mapping process depends on different parameters such as data transmit, zone type, segment and subchannel group [1]. The modulated data are serial to parallel converted (S/P) and then fed to the Inverse Fast Fourier Transform (IFFT) part, where each symbol is modulated by the corresponding subcarrier. Following the transformation process, the timed signal is serialized by using $\mathrm{P} / \mathrm{S}$ converter. To make the system more immune to the time selectivity of the channel, a guard time samples $\boldsymbol{v}$ is added as a cyclic prefix at the beginning of each transmitted OFDMA symbol. The signal samples are then passed through Digital to Analogue (DAC) converter then transmitted in a frame along with preamble, which used for channel estimation and synchronization. In the receiver side the, the received signal is first filtered, sampled and then serial to parallel converted. The guard time $\boldsymbol{v}$ samples are discarded and the remaining samples of each frame are demodulated by means of a FFT.

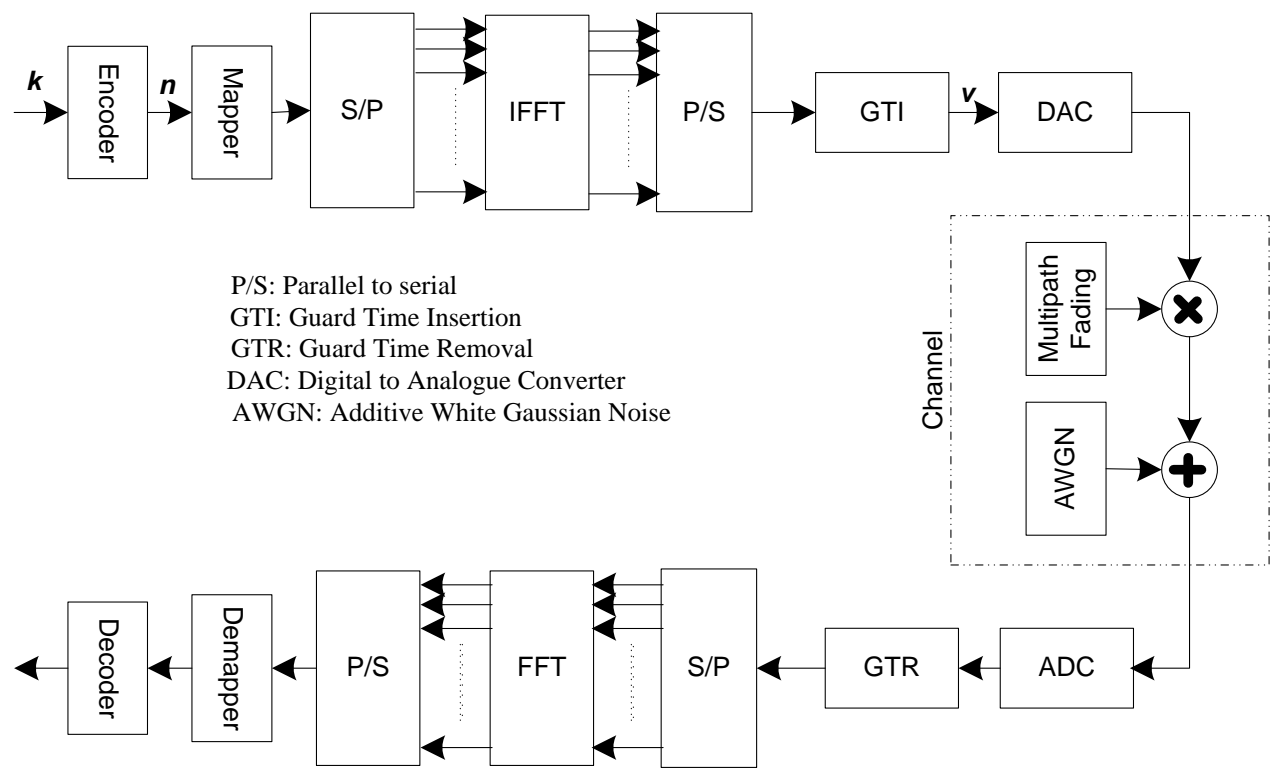

Figure 1: OFDMA Transceiver Block Diagram

\subsection{Guard Time Optimization}

A common rule of thumb used to select guard time span is to characterize the propagation channel delay spread. Practically GT span is either chosen two to four times of the maximum anticipated delay spread or kept $25 \%$ of the useful symbol time, which implies a $1 \mathrm{~dB}$ reduction in Signal to Noise Ratio (SNR). But it is still desirable to minimize the SNR degradation due to GT span [6]. However, in typical wireless mobile communication channel, mobile user expected to undergoes a wide range of operating conditions within short period of time or propagation distance. In such cases, the channel impulse response might be vary rapidly in some locations whereas in other vary slowly, with minimal delay spread. Based on that, fixing the GT span is impractical especially for mobile applications. This fact motivated the use of span guard time span [7]. On contrary adaptation requires a form of accurate either estimation or actual field measurements of channel delay spread. The later method are expensive, time consuming and unreliable. Thus it is crucial to find out an estimation technique that can quantify and update delay spread values wherever the mobile user is [8]. Some other related works have been reported as in [9] - [11].

\subsection{Delay Spread Estimation}

Time dispersion or delay spread introduces severe ISI at the receiver, which degrade the data transmission. Practically, its value found to be directly related to the propagation environment not on the system operating frequency [6]. Obviously, delay spread is not constant in wireless mobile communication channel. Measurements campaign [12] made in the U.S. covering wide range of geographies and topographical areas revealed that delay spreads are usually not exceeding 12 microseconds over measured locations. It was found that urban areas have RMS delay spreads on the order of 2-3 microseconds, about 5-7 microseconds in open and hilly residential areas, and high rise urban areas exhibit larger delay spreads in excess of 20 microseconds especially when the mobile traverses bridges. Measurements done by Seidel and Rappaport [13] in four 
European cities showed that delay spreads are less than 8 microseconds in macro-cellular channels, less than 2 microseconds in micro-cellular channels, and between 50 and 300 nanoseconds in pico-cellular channels. For indoor office building, the RMS delay spread is 35 nanoseconds, while at factory buildings, the delay spread goes up to 300nanoseconds [14].

In summary, depending on the terrain, distances, antenna directivity and other factors, the channel delay spread values can span from very small values (tens of nanoseconds) to large values (microseconds).

On the other hand, channel models defined by standard organization are heavily dependant especially when it is difficult to have an accurate description of the wireless channel. For example, ITU-R M.1225 [15] outdoor to indoor, pedestrian and vehicular channel models are baseline for design, development and testing of mobile 4G device.

\section{Calculation Example}

The following example shows how to calculate the GT, data rate and loss in SNR due to guard time insertion for mobile 4G system. Table 1 summarizes the primitive parameters that used for calculation and simulation works.

Table 1: Mobile 4G primitive parameters

\begin{tabular}{|l|l|}
\hline Parameters & Value \\
\hline Carrier frequency & $2300 \mathrm{MHz}$ \\
\hline System channel bandwidth $(B W)$ & $10 \mathrm{MHz}$ \\
\hline Sampling frequency $\left(F_{s}=\right.$ floor $\left.(n \times B W / 8000) \times 8000\right)$ & $11.2 \mathrm{MHz}$ \\
\hline FFTsize $\left(N_{F F T}\right)$ & 1024 \\
\hline Subcarrier frequency spacing $\left(\Delta f=F_{S} / N_{F F T}\right)$ & $10.9375 \mathrm{KHz}$ \\
\hline Useful symbol time $\left(T_{b}=1 / \Delta f\right)$ & $91.43 \mu S$ \\
\hline Guard time span $\left(G=T_{g} / T_{b}\right)$ & span \\
\hline Frame duration & $2 \mathrm{~ms}$ \\
\hline Modulation and coding & $16 \mathrm{QAM} 1 / 2$ \\
\hline Data subcarriers $\left(N_{\text {data }}\right)$ & 560 \\
\hline Pilot subcarriers & 280 \\
\hline Guard subcarriers & 184 \\
\hline & $91.1 \%$ \\
\hline
\end{tabular}

\subsection{Guard Time}

The OFDMA symbol consists of subchannels that carry data subcarriers carrying information, pilot subcarriers that are dedicated for synchronization and channel estimation purposes, DC subcarrier and guard subcarriers to provide high inter-channel interference margin. To determine subcarrier spacing and useful symbol time, sampling factor $n$ is commonly set to be $8 / 7$ for OFDMA PHY, yield sampling frequency $F_{S}=11.2 \mathrm{MHz}$. In order to keep the subcarrier spacing fixed at $10.9375 \mathrm{KHz}$ across different channel bandwidth, scalability feature of OFDMA chooses 1024 FFT span with $10 \mathrm{MHz}$ occupied bandwidth. This implies $91.1 \%$ bandwidth efficiency, but this percentage varies for other sampling factors and channel bandwidths. Thus, $T_{b}$ is the inverse of the subcarrier spacing $\Delta f$. Then GT is $T_{g}=G \times T_{b}$, where $G$ is $\frac{T_{g}}{T_{b}}$ ratio. The choice of $G$ made according to the radio channel condition. Thus, OFDMA symbol time ( $\left.T_{S}\right)$ comprising guard time span $\left(T_{g}\right)$ and useful symbol span $\left(T_{b}\right)$, where $T_{s}=T_{b}+T_{g}$.

\subsection{Data Rate}

The goal of a communications system is to provide higher data rates to the end users while minimizing the probability of errors. As per IEEE 802.16e-2005 standard, the maximum transmission raw data rate can be obtained using:

$$
R=\frac{N_{d a t a} \times b \times \frac{k}{n}}{T_{g}+T_{b}}
$$

Where $b$ is the number of bits per symbol for the modulation being used, $N_{\text {data }}$ is number of used subcarriers for data transmission and $\frac{k}{n}$ is the overall coding rate given in Table1. Further, it is also useful to describe channel capacity in terms of spectral efficiency using 
$\eta=\frac{C}{B W} \quad($ bits $/ \mathrm{s} / \mathrm{Hz})$

It is clear that, by changing the guard time span from $3 \%$ of the symbol span to $25 \%$ decreases the amount of data transmitted significantly which make OFDMA guard time is basic parameters for data rate computations. Table 2 provides an optimistic data rates achieved as function of modulation, coding and guard time span taking into account that these values do not consider some overheads such as preambles and signaling messages present in every frame.

\subsection{SNR Loss}

While increasing GT span to resist ISI and ICI, the overall power efficiency degrades proportionally. In particular, the loss in $E_{b} / N_{o}$ at the transmitter side becomes:

$S N R_{\text {loss }}=-10 \log _{10}\left(1-\frac{T_{g}}{T_{g}+T_{b}}\right)$

At the receiver, GT is removed before further processing, thus receiver energy remains unchanged. Table 2 shows the expected energy loss as function of GT. It can be noted that, however, minimizing power loss is needed because mobile terminals need to run on battery.

Table 2: OFDMA data rate and SNR loss.

\begin{tabular}{|c|c|c|c|c|}
\hline$T_{g}$ & \multicolumn{3}{|c|}{ Data rate (Mbps) } & \multirow{2}{*}{$\begin{array}{l}\text { Loss } \\
(\mathrm{dB})\end{array}$} \\
\hline & $\begin{array}{l}\text { QPSK } \\
1 / 2\end{array}$ & $\begin{array}{l}16 \mathrm{QAM} \\
1 / 2\end{array}$ & $\begin{array}{l}64 \mathrm{QAM} \\
3 / 4\end{array}$ & \\
\hline $1 / 4$ & 4.90 & 9.80 & 22.00 & 0.97 \\
\hline $1 / 8$ & 5.40 & 10.80 & 24.50 & 0.51 \\
\hline $1 / 16$ & 5.80 & 11.50 & 26.00 & 0.26 \\
\hline $1 / 32$ & 6.00 & 11.80 & 26.80 & 0.14 \\
\hline 0 & 6.20 & 12.30 & 27.60 & 0 \\
\hline
\end{tabular}

1. Computer Simulation

The performance of mobile 4G with span GT under multipath fading channel is evaluated using computer simulation.

\subsection{System Parameters}

The simulation parameters selected according to the IEEE 802.16e standard. As well, we chose the most relevant parameters that suit our local spectrum regularity. The parameters are; $10 \mathrm{MHz}$ nominal bandwidth, 1024 FFT size and $2.3 \mathrm{GHz}$ operating frequency. This spectrum is the most likely licensed band to roll out IEEE 802.16e services in Malaysia [MCMC]. To reduce the simulation time we use 2 ms frame duration instead of required $5 \mathrm{~ms}$. For efficient downlink (DL)/uplink (UL) asymmetric traffic support, the TDD duplexing mode is used with more than $60 \%$ of the frame time occupied by the DL subframe. The DL subframe uses Partially Used Subcahnnels (PUSC) zone type with maximum two number of symbols per slot. After preamble insertion, the FCH and DL_MAP are allocated. The former describes the subchannel used and transmission parameters. The later provides specific information about the DL bursts and their offsets.

For system performance evaluation, we chose 16 QAM modulation scheme and convolutional turbo coding (CTC) with overall coding rate 1/2. In particular, the ITU-R M.1225 channel model type A has been adopted to simulate mobile $4 \mathrm{G}$ for vehicular environment. The channel modeled multipath fading with six power decaying taps characterized by Ralyeigh distribution. The associated channel parameters are; 595 us RMS delay spread, $2510 \mathrm{~ns}$ maximum delay and $60 \mathrm{Km} / \mathrm{h}$ maximum speeds. The required bit energy per noise density $10 \mathrm{~dB}$ has been considered averaging over 1000 frames for probability of errors computation. In addition, the transmitted signal is corrupted by Additive White Gaussian Noise (AWGN) with noise density calculated as function of GT span using:

NDensity $=P_{S}-10 \log (1+G)-\frac{E_{b}}{N_{o}}-10 \log (R)$

Where $P_{s}$ signal power. The simulation assumptions for the evaluation are shown in Table1.

\subsection{Performance Evaluation}

When forward error correction techniques are used in wireless systems for packet data transmission on multipath fading channel, Packet Error Rate (PER) is a useful criterion for channel quality evaluation rather than Bit Error Rate (BER). 
To show the influence of varying the guard time span to the link quality for mobile $4 \mathrm{G}$ system, some simulation were made and the results are shown in Figure1: Simulation results for span guard time span. The GT implemented as Cyclic Prefix (CP) with various spans comprising; $\mathrm{CP}=0,0.0274,0.1625$ and 0.25 . Without $\mathrm{CP}$ systems are more susceptible to ISI, which is lower the data transmission rate. While with $25 \%$ of the symbol time spent on the CP, receivers are capable to collect longer multipath fading, but at the cost paid to SNR.

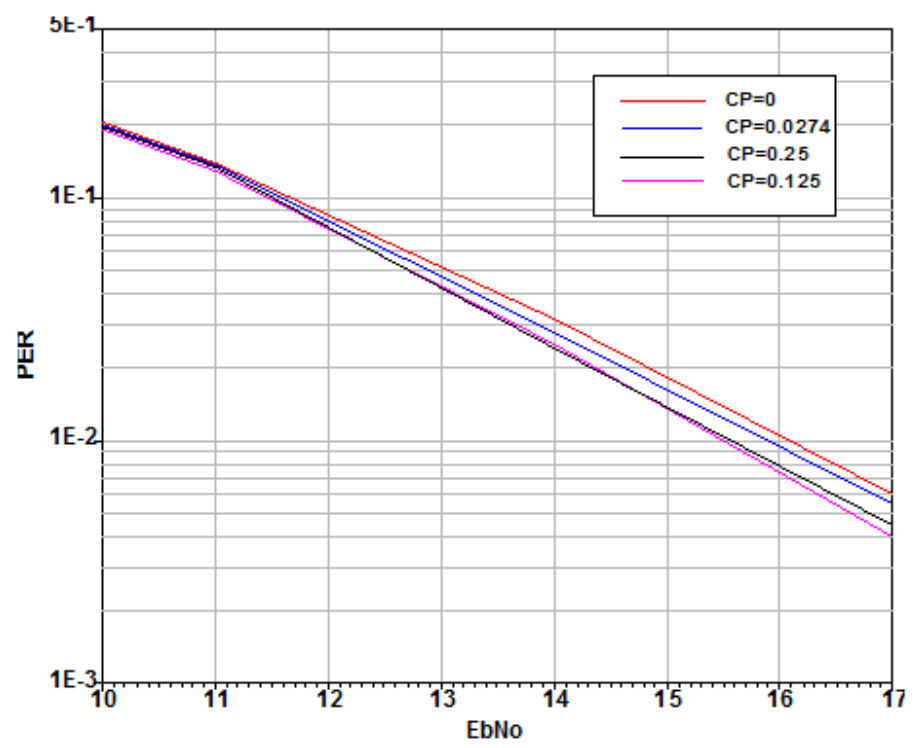

Figure 1: Simulation results for span guard time span.

From Figure1 it can be seen that PER does decrease as CP increase. The PER is plotted versus $\frac{E_{b}}{N_{o}}$

(the ratio of bit energy to noise power spectral density). It also shows that $\mathrm{CP}$ curves are almost identical below $11 \mathrm{~dB}$ and perform comparably beyond $15 \mathrm{~dB}$. It reveals that guard ratio of 0.125 slightly outperforms the other spans in PER reduction. In theory, the PER with $\mathrm{CP}=1$, over sufficient and impractical value, should be the same as that with 0.25 but the results are slightly different because they pass through diverse fading channels and the receiver is not ideal for them.

In fact, this behavior of the mobile receiver is expected when dealing with relatively small (RMS) delay spread comparing with one OFDMA symbol (2.746\%). Accordingly the effect of CP on system performance is also small. Consequently, it is mandatory to examine such a system under various channel models that have larger delay spread as in (channel model) for comparison and getting high system performance. Obviously, the optimal CP span is approximately 0.125 , a value that presently defined in mobile $4 \mathrm{G}$ profile.

\section{Conclusion}

Mobile 4G system performance as function of guard time span has been simulated under ITU-R vehicular channel model A. the results proves that guard time span is useful for OFDMA system operating on a frequency selective channel. Also it can be concluded, CP is strongly depends on maximum delay spread, not on RMS delay, and the optimal span found to be 1/8 of the OFDMA symbol time that minimize the degradation of SNR, caused by ISI. Lastly, varying guard time span is mandatory for mobile $4 \mathrm{G}$ to enhance overall system performance as well as to provide higher data rates.

\section{References}

[1] IEEE Std 802.16e-2005, IEEE Std 802. 2004/Cor 1-2005, "IEEE Standard for Local and metropolitan area networks, Part 16: Air Interface for Fixed and Mobile Broadband Wireless Access Systems, Amendment 2: Physical and Medium Access Control Layers for Combined Fixed and Mobile Operation in Licensed Bands and Corrigendum 1."

[2] 4G Forum. "4G's technology for LOS and NLOS Environments", 2004. [Online] Available: www.4Gforum.org.

[3] Hongwei Yang, A Road to Future Broadband Wireless Access: MIMO-OFDM-Based Air Interface, IEEE Communications Magazine, January 2005.

[4] Magnus Sandell, Jan-Jaap van de Beek, Per Ola Börjesson, "Timing and Frequency Synchronization in OFDM Systems Using the Cyclic Prefix", in Proceedings of the 1995 IEEE International Symposium on Synchronization, pp 16-19, Essen, Germany, December 1995.

[5] 4G Forum. "Mobile 4G - Part1: A Technical Overview and Performance Evaluation", August, 2006. [Online] Available: www.4Gforum.org.

[6] R. Van Nee and R. Prasad, OFDM for Wireless Multimedia Communications, Artech House, Boston, 2000.

[7] Das S. S., Fitzek F. and Prasad R, "Span Guard Interval OFDM in presence of carrier frequency offset", IEEE Global Telecommunication conference, GLOBECOM 2005, 28 Nov.- 2 Dec.2005, Vol. 5, on page(s): 5 pp. 
[8] H. Arslan and T. Yucek, "Delay Spread Estimation for Wireless Communication Systems", Proceedings of the Eighth IEEE International Symposium on Computers and Communication (ISCC'03).

[9] H. Steendam, M. Moeneclaey, "Guard Time Optimization for OFDM Transmission over Fading Channels", Proc. IEEE Fourth Symposium on Communications and Vehicular Technology SCVT'96, Ghent Oct 96, pp. 42-48.

[10] M. Bakir, M. Belhachat, J. G. Liu, S. Z. Zhu, "Optimization of Guard Interval for OFDM Performance over Fading and AWGN Channels Using Genetic Algorithm”, IEEE 6th CAS Symp. on Emerging Technologies: Mobile and Wireless Comm. Shanghai, China, May 31-lune 2,2004.

[11] Heidi Steendam, Marc Moeneclaey, Optimization of OFDM on Frequency-Selective Time-Selective Fading Channels, Signals, Systems, and Electronics, 1998. ISSSE 98. 1998 URSI International Symposium on 29 Sept.-2 Oct. 1998 Page(s):398-403

[12] T. S. Rappaport, S. Seidel, R. Singh, "900 MHz Multipath Propagation Measurements for U.S. Digital Cellular Radiotelephone," 1989 IEEE GLOBECOM Proceedings, Dallas, TX, November 29, 1989, pp. 8489

[13] Rappaport, T. S., and C.D. MaGillem, "Characterization of UHF Multipath Radio Channels in Factory Buildings" IEEE Transactions on Antennas and Propagation, Vol. 37, No.8, pp. 1058-1069, Aug. 1989.

[14] Scott Y. Seidel, Theodore S. Rappaport, Sanjiv Jain, Michael L. Lord, Rajendra Singh, "Path Loss, Scattering, and Multipath Delay Statistics in Four European Cities for Digital Cellular and Microcellular Radiotelephone", IEEE Transactions on Vehicular Technology, Vol.40, No.4, November 1991.

[15] ITU document Recommendation ITU-R M.1225, “Guidelines for Evaluation of Radio Transmission Technologies for IMT- 2000 Systems," 1998. 